\title{
Modular Bayesian Networks for Inferring Landmarks on Mobile Daily Life
}

\author{
Keum-Sung Hwang and Sung-Bae Cho \\ Dept. of Computer Science, Yonsei University \\ \{yellowg, sbcho\} @sclab.yonsei.ac.kr
}

\begin{abstract}
Mobile devices get to handle much information thanks to the convergence of diverse functionalities. Their environment has great potential of supporting customized services to the users because it can observe the meaningful and private information continually for a long time. However, most of the information has been generally ignored because of the limitations of mobile devices. In this paper, we propose a novel method that infers landmarks efficiently in order to overcome the problems. It uses an effective probabilistic model of Bayesian networks for analyzing various log data on the mobile environment, which is modularized to decrease the complexity. The proposed methods are evaluated with synthetic mobile log data generated.
\end{abstract}

\section{Introduction}

The mobile environment has very different characteristics from the ordinary personal computer environment. First of all, a mobile device can collect and manage various data about user. Besides, a mobile device can be adapted to fit user's preference or character since it is very private apparatus. Furthermore, a mobile device can collect everyday information effectively because a user brings it with him nearly every time, so it has a great potential to figure out and help its user. Such features of mobile device open the possibility of diverse and convenient services for user. However, there are some limitations of mobile devices. It has relatively insufficient memory capacity, lower CPU power (data-processing speed), and limited battery hours to desktop PC.

In this paper, we propose a novel method for effective analysis of mobile log data and extraction of semantic information and memory landmarks which are used as a special event for helping recall it [1]. The proposed method adopts Bayesian probabilistic model to efficiently manage various uncertainties occurring on mobile environment. We also propose a cooperative reasoning method for the modular model of Bayesian networks to work competently in mobile environments.

There already exist some attempts for analyzing log data and supporting expanded services. A. Krause, et al. clustered sensor and log data collected on mobile devices, learned a context classifier that reflected user's preference, and estimated user's situation to provide smart services to the user [2]. E. Horvitz, et al. proposed a method that detected and estimated landmarks by learning human's cognitive activity model from PC log data based on Bayesian approach [1]. However the models were based on the general learning method only for the small domain or for the PC domain. 


\section{Cooperative Reasoning of Modular Bayesian Networks}

The overall process of landmark extraction from log data of mobile environments used in this paper is shown in Fig. 1. Various log data are preprocessed in advance, and then the landmark reasoning module detects the landmarks. The preprocessing module is operated by the techniques of simple rule reasoning and pattern recognition. The $\mathrm{BN}$ reasoning module performs complicated and probabilistic inference.

Bayesian network is a model that can express large probability distributions with relatively small cost to statistical mechanics. It has a structure of directed acyclic graph (DAG) that represents link relations of node, and has conditional probability tables (CPTs) that is constrained by the DAG structure [3].

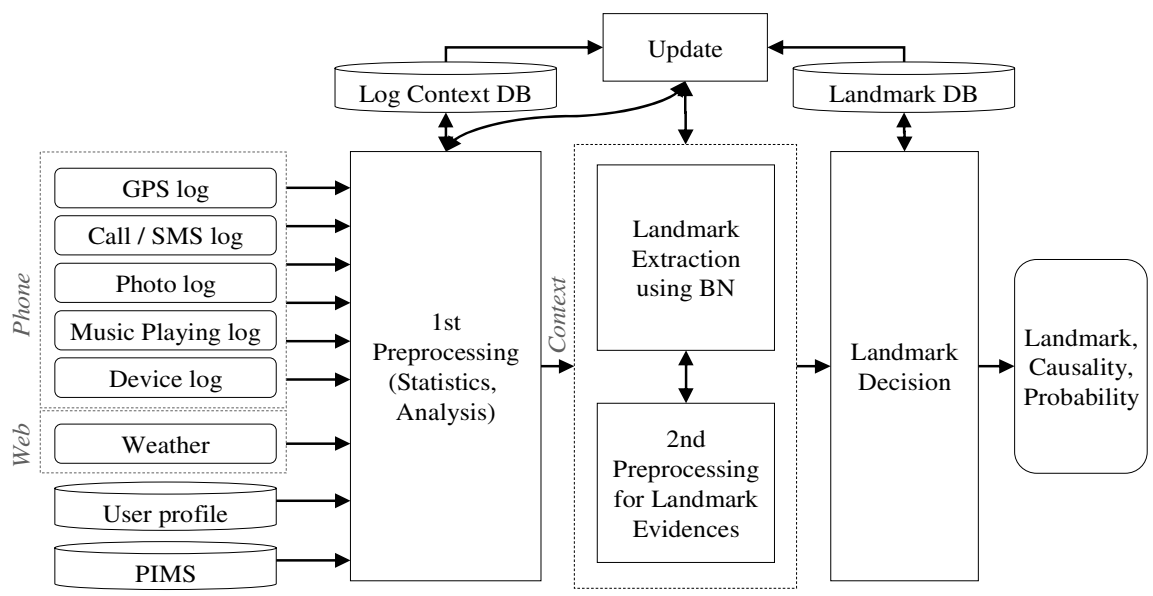

Fig. 1. The process of the landmark extraction from mobile log data

There are two general differences between the proposed Bayesian network and the conventional Bayesian network. Firstly, we modularize the Bayesian inference models according to their separated sub-domains (Fig. 2). Bayesian network model essentially requires more computing powers depending on the number of nodes and links. Especially, since the computational complexity of Bayesian inference is approximately proportional to $O\left(k^{N}\right)$, where $k$ is the number of states and $N$ is the number of causal nodes, the modularized $\mathrm{BN}$ is more efficient.

Secondly, in order to consider the co-causality of modularized BN, the proposed method operates 2-pass inference stages as shown in Fig. 2. A virtual linking technique is utilized in order to reflect the co-causal evidences more correctly. The technique is to add virtual node and regulate its conditional probability values (CPVs) to apply the probability of evidence [3]. In this paper, we have proposed and used the new virtual linking method that uses the prior probability of evidence node as virtual evidence parameter instead of adding virtual nodes. This method guarantees the maintenance of $\mathrm{BN}$ structure. For example, the structure of $\mathrm{BN}_{1}$ is $\{\mathrm{A} \rightarrow \mathrm{B} \rightarrow \mathrm{C}\}, \mathrm{BN}_{2}$ is its modularized version; $\{\mathrm{A} \rightarrow \mathrm{B}, \mathrm{B} \rightarrow \mathrm{C}\}$, and given the evidence $A$, we can calculate the beliefs using the virtual link assumption and chain rule as follows [3]: 


$$
\begin{gathered}
\mathrm{BN}_{1} \text { 's } \operatorname{Bel}(C)=P(A, C)=P(C \mid B) P(B \mid A) P(A) \\
\mathrm{BN}_{2} \text { 's } \operatorname{Bel}(B)=P(A, B)=P(B \mid A) P(A) \\
\mathrm{BN}_{2} \text { 's } \operatorname{Bel}(C)=P(B, C)=P(C \mid B) P(B)=P(C \mid B) P(A, B)=P(C \mid B) P(B \mid A) P(A) \\
\therefore \mathrm{BN}_{2}{ }^{\prime} \operatorname{sBel}(C)=\mathrm{BN}_{1}{ }^{\prime} \operatorname{Bel}(C)
\end{gathered}
$$

where the proposed virtual link assumption is $P(B)=\operatorname{Bel}(B)$.

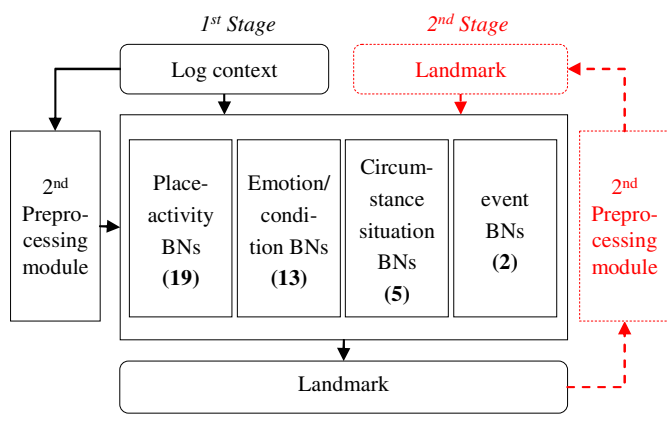

(a)

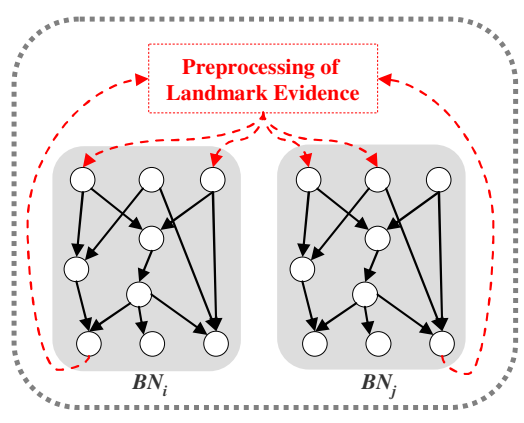

(b)

Fig. 2. (a) The 2-pass inference process for the cooperation of modular BNs. It uses the result from inference of $\mathrm{BN}$ as the evidences at the $2^{\text {nd }}$ inference stage. The dotted line indicates the stream of the $2^{\text {nd }}$ stage of inference processing. The parenthesis indicates the number of BNs contained. There are four kinds of BNs, and totally 39 BNs are used. (b) The modularized BNs for efficiency. The dotted line indicates a virtual link.

In this paper totally 39 BNs are designed with 638 nodes, 623 links and 4,205 CPV, which are summarized in Table 1. In average 16.6 nodes and 107.8 CPVs are used at the same time on the $\mathrm{BN}$ reasoning process since they are modularized and the computations are distributed. On the other hand, the monolithic $\mathrm{BN}$ for them has 462 nodes decreased from 638 because of the removal of duplicated nodes, but the number of parents and CPVs is increased. This means that it has much larger complexity.

Table 1. The information about the 39 modular BNs and the monolithic BN for them. Abbreviations: MonoBN - the monolithic BN corresponding to the modular BNs, NN - no. of nodes, NNR - no. of root nodes, NNI - no. of intermediate nodes, NNL - no. of leaf nodes, NL - no. of links, $\mathrm{NP}_{\mathrm{avg}}$ - avg. no. of parents, NS - no. of states, $\mathrm{NS}_{\mathrm{avg}}-$ avg. no. of states, NCPV - no. of CPVs, $\mathrm{CPT}_{\max }-$ maximum size of CPT.

\begin{tabular}{lllllllllll}
\hline & NN & NNR & NNI & NNL & NL & NP $_{\text {avg }}$ & NS & NS $_{\text {avg }}$ & NCPV & CPT $_{\max }$ \\
\hline 39 BNs & 638 & 375 & 135 & 128 & 623 & 0.98 & 1,279 & 2.00 & 4,205 & 64 \\
MonoBN & 462 & 235 & 111 & 116 & 588 & 1.27 & 927 & 2.01 & 4.869 & 512 \\
\hline
\end{tabular}

\section{Experimental Results}

We have tested the proposed landmark reasoning model with a scenario in order to confirm the performance. The left side of Fig. 3 shows a scenario used. The BN set 
strongly related to the scenario is ffood, photo, movement, nature, joy, home . The probabilities are occurred when the related evidences are given. The right side of Fig. 3 shows the inference results. We can see the increment of the probabilities of the related landmarks at the corresponding time. For example, there are 'going-outpreparation' and 'shower' landmarks at 7 9 o'clock, 'eating' at 12 13 and 17 19 o'clock, 'walking-for' at 13 14 and 20 21 o'clock, 'joyful-photo' at 14 15 o'clock, and 'eating-out' and 'eating (western style)' landmarks at 17 19 o'clock.

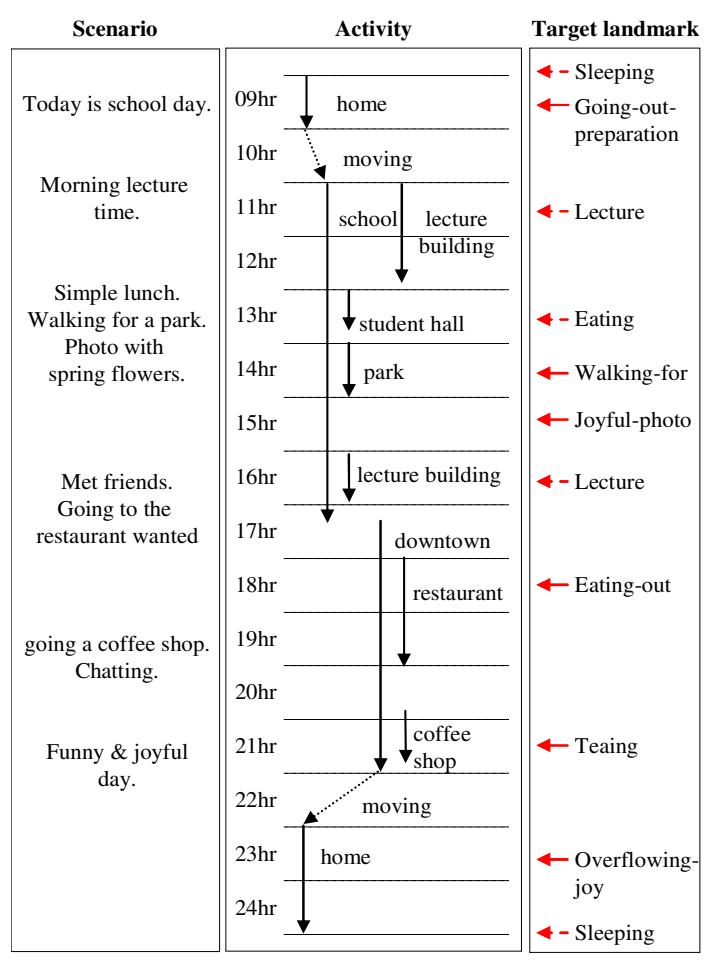

(a)

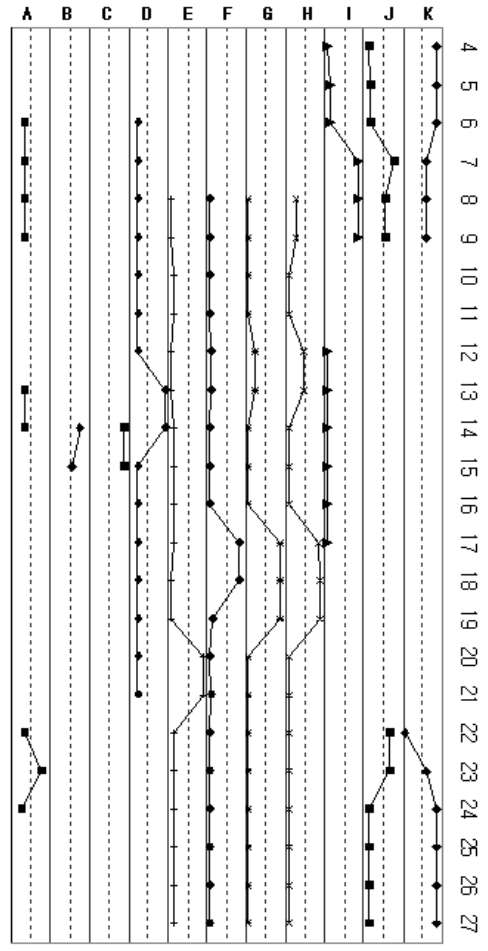

(b)

Fig. 3. (a) A scenario of an everyday life with mobile device of an undergraduate student for experiments. (b) The probability values of 11 target landmarks. The denoted time is from 4 o'clock to 27 o'clock (equal to 3 o'clock tomorrow). Abbreviations used for landmarks: A Overflowing-joy, B - Photo (scenery), C - Joyful-Photo, D - Walking-for, E - Tea, F - Eatingout, G - Eating (western style), H - Eating, I - Going-out-preparation, J - shower, K - sleeping.

We grouped situations of everyday life on the two bases: usual/unusual and idle/busy. We made contexts for evidence because it is difficult to make the log data directly. For example, we made a context 'a lot of phone calls' instead of its phone call $\log$ data. The target landmarks are categorized 4 classes as shown in Table 2. A day of data set contains two landmarks, which are selected randomly. Each landmark has some candidates of evidence set and one of them is selected randomly. For example, the landmark 'annoying SMS' has candidates, \{'a lot of phone calls', 'a lot of moving' $\},\{$ 'a lot of moving', 'a lot of SMS'\}, \{'a lot of phone calls', 'a lot of SMS'\}. 
Table 2 shows the statistics of experimental results. We have excluded the landmarks related to the default place 'home' and the low-weight landmarks from main landmarks set. The false-positive error of 'usual/idle' class is high and the precision is low, because 'usual' class includes many places and landmarks that have evidence duplication. For example, since the landmark 'boarding ship' is caused by the evidence 'sea' or 'river', a landmark 'swim' can also be extracted. The false-positive error of 'usual/busy' class is low because the class includes relatively many landmarks that have distinct evidence. In the experiment, the overall recall rate was low as $75 \%$. It results from the lack of tuning or the landmarks hard to detect.

Table 2. The experimental results with synthetic data. Two target objects (with few redundancies) are selected in each data set. 'unusual/busy' class data are composed of one 'unusual' landmark and one 'busy' landmark. Abbreviations: LM-Landmark, TP-true positive error rate $(\%)$, FP-false positive rate (\%), $\mathrm{FN}$-false negative rate (\%), \#-number.

\begin{tabular}{cccccccc}
\hline Class & Time & Target LMs \# & TP & FP & FN & Precision & Recall \\
\hline usual/idle & 30 days & 60 & 46 & 14 & 14 & 0.767 & 0.767 \\
unusual/idle & 30 days & 58 & 43 & 10 & 15 & 0.811 & 0.741 \\
usual/busy & 30 days & 55 & 41 & 2 & 14 & 0.953 & 0.745 \\
unusual/busy & 30 days & 60 & 46 & 8 & 14 & 0.852 & 0.767 \\
\hline Total & 120 days & 233 & 176 & 34 & 57 & 0.838 & 0.755 \\
\hline
\end{tabular}

\section{Concluding Remarks}

In this paper, we have proposed a landmark inference model for it to be more suitable to mobile device environment. We have modularized BN structures for more efficient operation in mobile environment and proposed 2-step inference method of applying virtual node concept for cooperation of modular BNs. In some experimental results with artificial data, the intended landmarks are well extracted. Currently, this research is ongoing, and we will validate the performance and utility of the proposed method on the expanded real-world domain in the future.

Acknowledgement. This research was supported by the Ministry of Information and Communication, Korea under the Information Technology Research Center support program supervised by the Institute of Information Technology Assessment, IITA2005-(C1090-0501-0019).

\section{References}

[1] E. Horvitz, S. Dumais, and P. Koch. "Learning predictive models of memory landmarks," CogSci 2004: 26th Annual Meeting of the Cognitive Science Society, pp. 1-6, 2004.

[2] A. Krause, A. Smailagic, and D.P. Siewiorek, "Context-aware mobile computing: Learning context-dependent personal preferences from a wearable sensor array," IEEE Trans. on Mobile Computing, vol. 5, no. 2, pp. 113-127, 2006.

[3] K.B. Korb and A.E. Nicholson, Bayesian Artificial Intelligence, Chapman \& Hall/CRC, 2003.

[4] M. Raento, A. Oulasvirta, R. Petit, and H. Toivonen, "ContextPhone: A prototyping platform for context-aware mobile applications," IEEE Pervasive Computing, pp. 51-59, 2005. 